EPJ Web of Conferences 19, 06005 (2012)

DOI: $10.1051 /$ epjconf/20121906005

(C) Owned by the authors, published by EDP Sciences, 2012

\title{
Towards the big picture of the Milky Way bulge
}

\author{
O.A. Gonzalez
}

European Southern Observatory, Karl-Schwarzschild-Strasse 2, 85748 Garching, Germany

\begin{abstract}
Evidence has been growing in the last few years that the Bulge is very complex, with maybe two or more components partially overlapping in space, kinematics and metallicity. An extensive mapping of these properties is necessary to disentangle them. I present our results based on $\alpha$-elements abundances for a total of 650 RGB stars in 4 fields along the major and minor axis of the Bulge. Results point towards a spatial homogeneity of $\alpha$-element enhancement in the metal-poor regime and a population of alpha-poor metal-rich stars which disappears at high latitudes $(b=-12)$. Bulge metal-poor alpha enhancement is also indistinguishable from the one of the thick disk. Further constrains on Bulge populations are provided by the Vista Variables in the Via Lactea survey (VVV) from which I present our recent results in extinction, structure and photometric metallicities.
\end{abstract}

\section{INTRODUCTION}

The Milky Way bulge provides an important laboratory to investigate the imprints of how the Galaxy formed and evolved. Because of extinction limitations, early studies of the bulge were restricted to Baade's window, at a latitude $b=-4^{\circ}$ along the minor axis. More recently, several papers have presented evidences of a high complexity in the bulge structure and stellar population content. In terms of the observed chemical signatures of its stars, the Milky Way bulge shows a radial metallicity gradient along the minor axis [1,2] that most likely flattens in the inner regions [3], which might be explained by the coexistence of two kinematically and chemically distinct components [4-7]. Furthermore, in terms of structure, the outer parts of the Bulge $(|b|>5)$ have been found to be consistent with a X-shape morphology [8]. All these observations strongly argue against a generalization of the stellar population properties observed in a few low-extinction windows of the Bulge. The need to investigate the extent of the chemical gradients, kinematical properties of its components and structure across different regions, is evident.

\section{THE ALPHA-ELEMENT ABUNDANCES FOR 650 STARS IN 4 BULGE FIELDS}

In [5] we used the sample analyzed in [1] as part of a project aimed to provide a spectroscopic characterization of the Galactic bulge. The sample consists of $650 \mathrm{~K}$ giant stars spectra, from 3 fields along the Bulge minor axis $(b=-4,-6,-12)$ and another field at $(l, b)=(-5,-4)$, obtained with the FLAMES-GIRAFFE spectrograph, using the HR13 setup $(\mathrm{R}=22$ 500). On the other hand, the sample for thick and thin disk RGB stars in the local neighborhood consists of the same spectra analyzed in [9]. Stellar parameters and abundances for $\mathrm{Ca}, \mathrm{Mg}$, Ti and $\mathrm{Si}$ were obtained in the exact same way for both samples, in order to perform a differential analysis as described in [5].

\footnotetext{
a e-mail: ogonzale@eso.org
}

This is an Open Access article distributed under the terms of the Creative Commons Attribution-Noncommercial License 3.0, which permits unrestricted use, distribution, and reproduction in any noncommercial medium, provided the original work is properly cited. 


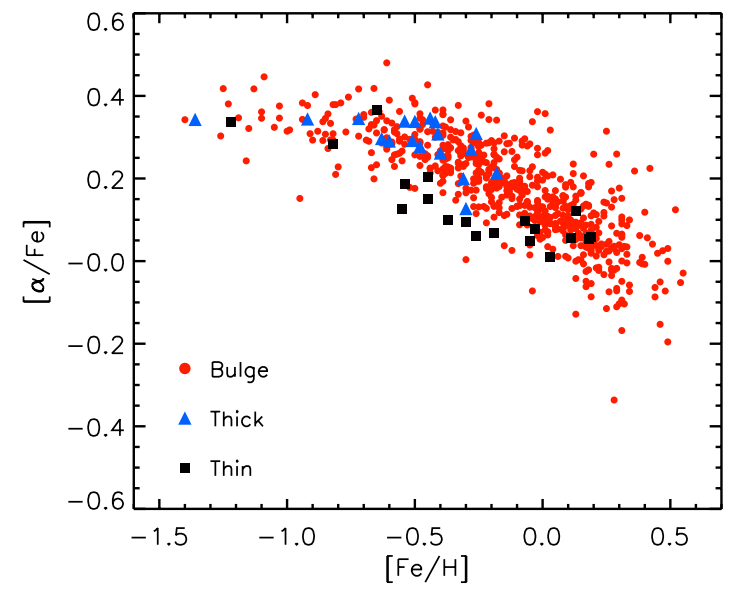

Figure 1. $[\alpha / \mathrm{Fe}]$ abundances calculated as the average between $\mathrm{Ca}, \mathrm{Mg}$, Ti and $\mathrm{Si}$ abundances for 650 bulge giant stars shown as red filled circles. Abundances for the thick disk stars are shown as blue filled triangles and as filled black squares for the thin disk stars.

Figure 1 shows the average $\alpha$-element abundances for the complete bulge and disk sample. The trends for bulge and disk populations are very well defined and, in particular, the similarity between the Bulge and thick disk is clearly observed. Indeed, when considering the mean abundances of all the elements, differences between the bulge and the thick disk do not exceed 0.05 dex for all metallicities. Additionally we see that the knee in the bulge trend, identified as the instance when the contribution of SNIa becomes important, is located at a metallicity of $[\mathrm{Fe} / \mathrm{H}]=-0.44$. This knee is also present in the thick disk distribution at $[\mathrm{Fe} / \mathrm{H}]=-0.51$, only 0.07 dex different to the location of the bulge downtrend. Therefore, within the errors considered in our analysis, trends between both populations are indistinguishable. This hints for a similar formation scenario for both the bulge and the thick disk.

Additionally, the left panel of Fig. 2 shows how the observed trends in alpha element abundances with metallicity, have no variations across the three fields along the minor axis. However, coupling the information from radial velocities, proper motions, and metallicity, for the same sample of Bulge spectra along the minor axis, [4] demonstrated that there is a metal-rich component which shows disk/bar kinematics and a metal-poor component with kinematics of an old spheroid. The old metalpoor spheroid component is expected to have a much shorter formation time-scale than the disk/bar component, and it is therefore expected to be more alpha enhanced. We investigate these expected differences in alpha element abundances by combining our abundance measurements with the radial velocity information. Figure 2 shows the radial velocity dispersion in bins of alpha element abundances. We observe that alpha-enhanced stars have an approximately constant velocity dispersion along the minor axis of $\sim 90 \mathrm{~km} / \mathrm{s}$, while the stars with solar or slightly sub-solar $[\alpha / \mathrm{Fe}]$ abundances have larger velocity dispersion in the inner fields and decreasing at higher latitudes. Furthermore, this alpha-poor component is no longer observed at a latitude of $b=-12$. Thus, the bulge hosts a metal-poor alphaenhanced component, which shows no variation, neither in abundances nor kinematics, among different bulge regions and, as shown before, is chemically indistinguishable from the thick disk. This hints for a fast and early formation for both the metal-poor bulge component and the thick disk. On the other hand, the metal-rich component with bar-like kinematics, shows low $[\alpha / \mathrm{Fe}]$ similar to those of the thin disk and is therefore expected to have formed on a more extended timescale.

\section{EXTINCTION AND METALLICITIES MAPS BASED ON VVV DATA}

The VISTA Variables in Via Lactea (VVV) is the Galactic near-IR ESO VISTA public survey that started collecting data in 2010. The VVV area covers two main components: the 300 sq. deg VVV Galactic 

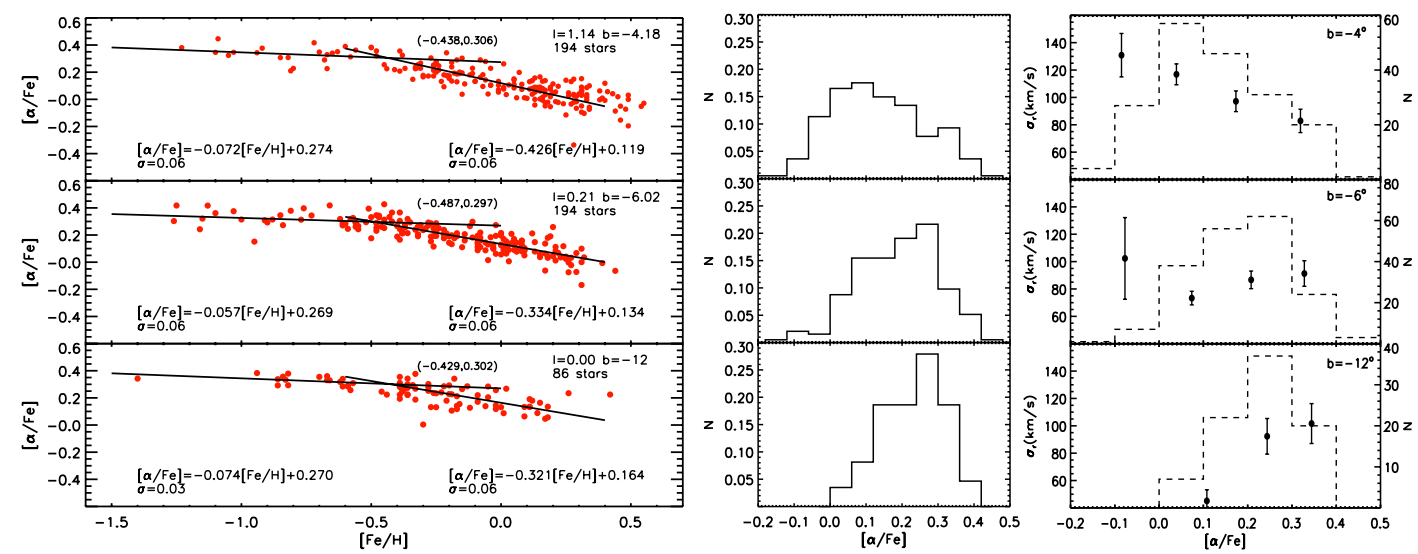

Figure 2. Left panel: $[\alpha / \mathrm{Fe}]$ trends as a function of $[\mathrm{Fe} / \mathrm{H}]$ in 3 bulge fields located along the minor axis, $b=-4$, -6 and -12 . Best fit trends are shown for both $[\mathrm{Fe} / \mathrm{H}]$ ranges, a metal-poor between -1.2 and -0.5 dex and a metalrich between -0.3 and 0.2 dex, as well as the location of the knee in all fields. Center panels: $[\alpha / \mathrm{Fe}]$ distribution for each field. Right panel: Dispersion of radial velocity as a function of $[\alpha / \mathrm{Fe}]$, in bins of $0.15 \mathrm{dex}$, for the three fields along the minor axis (filled circles and left axis scale). Overploted as a dashed line is the $[\alpha / \mathrm{Fe}]$ distribution for each field, which scale is given on the right.

bulge survey area covering $-10^{\circ}<l<+10^{\circ}$ and $-10^{\circ}<b<5^{\circ}$, and the 220 sq. deg VVV plane survey area spanning from $295^{\circ}<l<350^{\circ}$ to $-2^{\circ}<b<2^{\circ}$. These areas will be imaged repeatedly in $K_{s}$-band with the aim to search for variable stars such as RR Lyrae in the Milky Way bulge. In addition to the main variability campaign, the survey area is fully imaged in five photometric bands: $\mathrm{Z}, \mathrm{Y}, \mathrm{J}, \mathrm{H}$, and $K_{s}$. Here I summarize the method and results presented in [10] based on the single-epoch JHKs coverage of the bulge. The more complete description of the whole survey and the observation strategy is available in [11].

In order to test a method to obtain reddening using VVV information towards the bulge, we used the $\left(J-K_{s}\right)$ colors of red clump (RC) giants along the bulge minor axis, between $b=-8^{\circ}$ and $b=-0.5^{\circ}$, at longitudes between $0^{\circ}$ and $2^{\circ}$. The area was split into 1835 sub-fields with boxes of $3^{\prime}$ on a side for fields within inner $1.5^{\circ}, 6^{\prime}$ for $-5<b<-1.5^{\circ}$, and $10^{\prime}$ for $b<-5^{\circ}$, for which we obtained the extinction $\mathrm{E}(\mathrm{B}-\mathrm{V})$, by comparison to that of Baade's window. We measured the color of the red clump in Baade's Window, a $10^{\prime} \times 10^{\prime}$ region centered at $l=1.14$ and $b=-4.18$ for which we adopted a reddening value of $\mathrm{E}(\mathrm{B}-\mathrm{V})=0.55$ as in [1]. In the observed $\left(J-K_{s}\right)$ color histogram of this region we measured a mean value of $\left(J-K_{S}\right)_{R C}^{B W}=0.96$ based on a Gaussian fit to the color distribution which is compared to each one of the sub-fields in our bulge region in order to obtain the corresponding reddening value. Our procedure allows us to obtain dereddened $K_{s}$ magnitudes while keeping the imprints of the original bulge stellar population. Because of its broad metallicity distribution, a single intrinsic RC color should not be adopted for all stars but only to the mean of the observed RC color distribution in small sub-fields where the effects of differential extinction are minimized. The middle panel of Fig. 3 shows the reddening map obtained for the bulge minor axis. The small-scale variations are clearly observed and they become particularly strong in the inner regions. This is evident in the left panel of Fig. 3 which shows the reddening map for $b>-1.4$. As expected, this indicates that the use of lower resolution maps for the very inner bulge regions, near to the Galactic plane, will not be sensitive to such variations.

We then applied a color cut $\left(J-K_{s}\right)_{0}>0.4$ to select the region of the color-magnitude diagram dominated by bulge giants and avoid contamination from disk stars. Using the dereddened $K_{s_{0}}$ magnitudes of bulge stars, we built the luminosity function for each tile and measured the RC mean magnitudes, using a Gaussian fitting procedure, in order to trace the mean distance to the bulge population in each particular line of sight. The base of the luminosity function was fitted with a second 


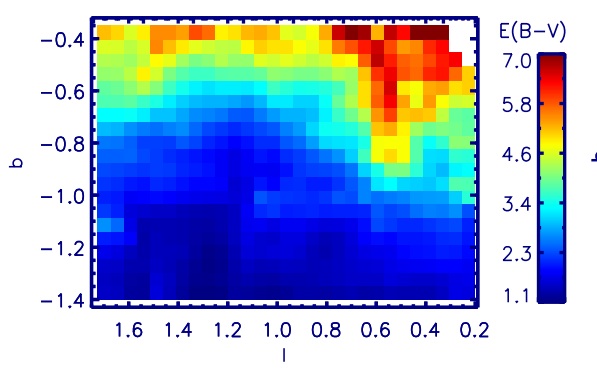

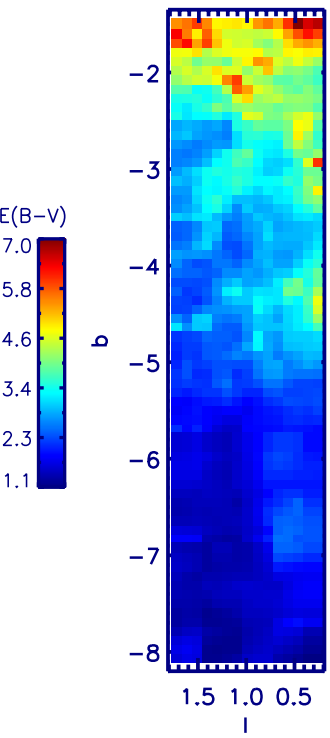

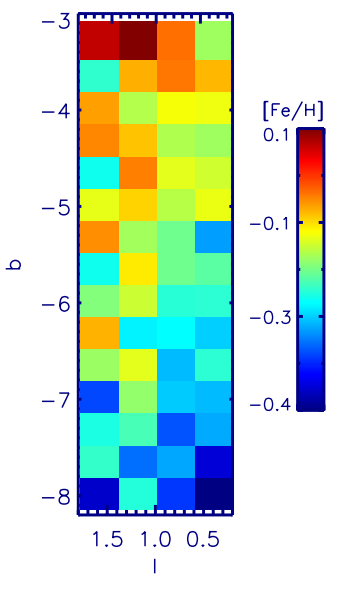

Figure 3. Left panel: Reddening map for the inner regions of the bulge along the minor axis. Center panel: Reddening map along the minor axis of the bulge. Regions with $b<-5$ are smoothed to $6^{\prime}$ boxes for visualization. Right panel: Map of the mean values of the metallicity distributions along the bulge minor axis for sub-fields of $\sim 0.4 \times 0.4$.

order polynomial and a Gaussian fit was then applied to measure the RC mean magnitude. We then used the observed mean RC magnitudes to obtain distances for each of our sub-fields through a comparison of these measured values with the intrinsic RC magnitude for a $10 \mathrm{Gyr}$ solar metallicity population of $\mathrm{M}_{K_{\mathrm{s}}}=-1.55$.

We compared the derived CMDs in each subfield, now in the $\left[\mathrm{M}_{K_{s}},\left(J-K_{s}\right)_{0}\right]$ absolute plane owning to the derived distances using the RC, with an empirical grid of RGB ridge lines of globular clusters. This allows us to derive a metallicity value for each field RGB star based on its $\left(J-K_{s}\right)_{0}$ color, by interpolation within the empirical templates of Galactic halo and bulge globular clusters, with well known metallicity, selected from the sample of [12]. The derived individual metallicities were used to build the metallicity distributions in each subfield. The right panel of Fig. 3 shows a map of mean metallicity in bins of $\sim 0.4^{\circ} \times 0.4^{\circ}$ which confirms that the metallicity gradient along the minor axis can be traced with our method. Furthermore, as shown in [10], the shape of the photometric distributions and mean values resemble those from spectroscopic studies very closely. These maps are restricted to $|b|>3^{\circ}$ as the upper RGB in our dataset corresponds to 2MASS photometry, which is limited by crowding at inner regions. Altogether, this presents a powerful method to trace extinction, structure and metallicity gradients across the Bulge. In a future paper we will present the extinction maps of the entire VVV bulge area and metallicities for fields with latitudes $|b|>3^{\circ}$.

\section{References}

[1] M. Zoccali, V. Hill, A. Lecureur, et al., A\&A, 486, 177, (2008)

[2] C. I. Johnson, R. M. Rich, J. P. Fulbright, et al., ApJ, 732, 108, (2011)

[3] R. M. Rich, L. Origlia \& E. Valenti, ApJ, 665, L119, (2007)

[4] C. Babusiaux, A. Gomez, V. Hill, et al., A\&A, 519, A77, (2010)

[5] O. A. Gonzalez, M. Rejkuba, M. Zoccali, et al., A\&A, 530, A54, (2011)

[6] V. Hill, A. Lecureur, A. Gomez, et al., A\&A, 534, A80, (2011) 
[7] T. Bensby, D. Aden, J. Melendez, A\&A, 533, A134, (2011)

[8] A. McWilliam \& M. Zoccali, ApJ, 724, 1491, (2010)

[9] A. Alves-Brito, J. Melendez, M. Asplund, et al., A\&A, 513, A35, (2010)

[10] O. A. Gonzalez, M. Rejkuba, M. Zoccali, et al., A\&A 534, A3, (2011)

[11] D. Minniti, P. W. Lucas, J. P. Emerson, et al., New A, 15, 433, (2010)

[12] E. Valenti, F. R. Ferraro, \& L. Origlia, MNRAS, 351, 1204, (2004) 